\title{
Re-conceptualizando el desarrollo humano: Universidad y Sostenibilidad
}

Re-conceptualizing human development: University and Sustainability

\author{
Belén Sáenz-Rico de Santiago ${ }^{1 凶}$ \\ ${ }^{1}$ Universidad Complutense de Madrid (España) \\ ${ }^{凶}$ Autor para correspondencia: $\underline{\text { bsaenzri@edu.ucm.es }}$
}

Recibido: $15 / 04 / 2019$

Aceptado: 15/05/2019

\begin{abstract}
Otros mundos, en este
En este caso, y en alusión implícita a lo que escribió el poeta Paul Eluard: el “otro"mundo, los otros mundos, están aquí, en el mundo en el que vivimos.

Se trata de dirigir nuestra mirada hacia aquello que habitualmente no vemos, aunque lo tengamos ante nuestros ojos.
\end{abstract}

\section{CONTENIDO}

Nadie se atrevería a poner en duda que estamos atravesando una profunda crisis. Desde algunas perspectivas, ésta se pone de manifiesto en la subida de la prima de riesgo, la caída de la bolsa, el aumento del déficit público, el desequilibrio en la balanza de pagos o la pérdida de la confianza de los mercados. Para otros, la crisis es más que la alteración de unos indicadores que describen la coyuntura económica. La crisis del sistema se evidencia por la crisis ambiental, la crisis de derechos humanos políticos, sociales, culturales, económicos, etc. que generan exclusión y que afectan, aunque de diferente forma, al conjunto del planeta.

Nadie pone en duda que el ser humano ha conseguido impactar en el sistema tierra, de tal forma que en el mundo científico se discute en la actualidad la definición de una nueva era geológica llamada Antropoceno. Los sistemas sociales, económicos, políticos y culturales han desestructurado los sistemas naturales, superando su capacidad de resistencia y adaptación. Poniendo en serio peligro el equilibrio dinámico de todos ellos.

Esta crisis ecológica global es ante todo una crisis civilizatoria en la que hay implicados valores, ideas, perspectiva y conocimiento (Orr, 1994); esto es, una crisis de educación, aunque no solamente en la educación.

Si ampliamos el alcance de nuestra mirada, nos hacemos conscientes de que, según los últimos datos de la Organización de las Naciones Unidas para la alimentación y la agricultura (FAO), el número de personas desnutridas tras haber disminuido de forma constante durante más de una década, volvió a aumentar en 2016, el $11 \%$ de la población mundial, lo que afectó a 815 millones de personas. En Asia a 520 millones; 243 millones en África y 42 millones en Latinoamérica y el Caribe.

Según el informe anual de la ONU (2017) sobre seguridad alimentaria y nutrición este incremento de 38 millones de personas más respecto al año anterior, se debe en gran medida a la proliferación de conflictos violentos y de perturbaciones relacionadas con el clima. Unos 155 millones de niños menores 
de cinco años padecen desnutrición crónica, según el informe, mientras que 52 millones sufren de desnutrición aguda, lo que significa que su peso es demasiado bajo para su estatura. Se estima además que 41 millones de niños tienen sobrepeso. La anemia en las mujeres y la obesidad adulta son también motivo de preocupación. Cifras que son fruto de la especulación con el precio de los alimentos, el cambio climático y el auge de los agrocombustibles, presentados en nuestras sociedades como el sustituto al petróleo y, en consecuencia, la garantía del mantenimiento de nuestro modelo de desarrollo y nuestro nivel de vida.

El $75 \%$ de las variedades Genéticas de los cultivos agrícolas han desaparecido en el último siglo a consecuencia de una lógica de monocultivos y monopolios que se traduce en alimentos de mala calidad, a bajos precios para los consumidores y consumidoras de los países del Norte, hambre y miseria en los países empobrecidos y grandes beneficios para los intermediarios y las empresas multinacionales del sector.

El 95\% de la soja cultivada en el mundo se destina a la elaboración de piensos para ganadería con el fin de satisfacer la demanda de productos cárnicos en Europa y Norteamérica; así como a la elaboración de agro-combustibles. En Argentina, Brasil y Paraguay se cultivan semillas genéticamente modificadas y se utiliza masivamente el glifosato, un agrotóxico que causa la deforestación de vastas extensiones de terreno, responsables de $31.7 \%$ de los gases de efecto invernadero. El uso generalizado y cada vez más intensivo del glifosato en asociación con el uso de cultivos GM (genéticamente modificados, también denominados transgénicos) plantea riesgos adicionales para el medio ambiente y la salud humana. El uso del glifosato en cultivos transgénicos RR tales como la soja, el maíz y el algodón, se ha incrementado drásticamente en toda América, donde predomina su cultivo (Greenpeace, 2011)

Lo irónico es que nunca como en nuestros días se han producido en el mundo tantos alimentos $\mathrm{y}$, sin embargo, el $70 \%$ de las personas que pasan hambre son quienes la producen.

Estamos en un periodo de desequilibrio e inestabilidad mundial, ¿crisis? ¿Por qué se caracteriza?. La crisis que se nos presenta en la actualidad comenzó hace apenas unos pocos años, y afecta a algunos países que hasta ahora se habían percibido a sí mismos como los países desarrollados. Sin embargo, la desigualdad, la exclusión y la vulneración de derechos humanos no conocen fronteras, y no sólo se vienen produciendo desde hace décadas, sino más bien, que en los últimos años se han visto agravadas.

El cambio climático ocasiona sequías inusuales e inundaciones que destruyen tierras y cosechas, por lo que se prevé una reducción de la productividad de cultivos importantes para la alimentación humana. A nivel global, el cambio en las precipitaciones y la desaparición de los glaciares también podría afectar al acceso al agua potable.

Como vemos, la crisis nos pone en relación: sus consecuencias afectan a toda la humanidad, todos y todas podemos encontrar a la vez nuestra parcela de responsabilidad en ella. La crisis en la que nos encontramos no se reduce, por lo tanto, al colapso financiero. Es una crisis global, acumulada y multidimensional, es la crisis del modelo de desarrollo dominante (Fernández, 2011).

Paremos en este momento y reflexionemos de manera individual sobre el engranaje que mueve el planeta: Permítanme que mi reflexión la realice en voz alta.

Para el sistema dominante, el capitalismo, es la acumulación de capital lo que impulsa el desarrollo, transformando la lógica que existía previamente según la cual los mercados 
permitían sencillamente el intercambio de bienes y servicios entre quienes accedían a él para satisfacer sus necesidades. El capitalismo cambia el sentido de la actividad económica, es decir, produce mercancías, no para intercambiarlas y conseguir así otras mercancías, sino para hacer crecer el capital inicial.

En el primer caso, las mercancías podían tener un valor similar, pues el sentido del intercambio era la mejor satisfacción de las necesidades humanas. En el segundo caso, el sentido de la actividad es que el capital resultante sea mayor que el inicial, por lo que poco importa la utilidad de la mercancía.

La actividad del mercado inicial se relajaba en el momento en el que la satisfacción de necesidades era suficiente, pero en el momento actual, la actividad debe ser constante y siempre creciente, pues sólo el crecimiento permanente, la producción y el consumo constante, permiten la acumulación del capital. Para terminar de complicar y desconectar el mercado de las necesidades humanas, a lo largo del siglo XX hemos asistimos a la aparición de la economía especulativa, en la que ya ni siquiera es necesaria la producción de la mercancía o la prestación del servicio.

\section{"HASTA AQUÍ HEMOS LLEGADO”}

En 1972, el Informe "Los límites del crecimiento", publicado por el Club de Roma, afirmaba que:

"Si el actual incremento de la población mundial, la industrialización, la contaminación, la producción de alimentos y la explotación de los recursos naturales se mantiene sin variación, alcanzará los límites absolutos de crecimiento en la Tierra durante los próximos cien años".
El informe se apoyaba en la idea de que «en un planeta limitado, las dinámicas de crecimiento exponencial no son sostenibles» $\mathrm{y}$, en la actualidad, podemos afirmar que tanto el crecimiento como la destrucción de la naturaleza ha sido más veloz de lo que entonces previeron. Como consecuencia de un consumo creciente de materia y energía nos estamos acercando al pico de distintas materias prima de las que nos hemos y nos han hecho dependientes, como el petróleo. A partir de este pico solo podrá ocurrir una cosa, máxime en un entorno de demanda creciente como el actual, y es que el precio se disparará hasta hacerse inasumible económicamente o hasta su agotamiento. En los últimos tiempos estamos viendo el aumento de conflictos bélicos para esta materia prima, encubiertos en postulados culturales-religiosos con el único objetivo de dominar el mundo.

En la última década, el número de conflictos ha aumentado de forma dramática y se han vuelto más complejos e irresolubles por su naturaleza, afirman los responsables de la Organización de las Naciones Unidas para la Alimentación y la Agricultura (FAO), el Fondo Internacional de Desarrollo Agrícola (FIDA), UNICEF, el Programa Mundial de Alimentos (PMA) y la Organización Mundial de la Salud (OMS). Subrayan que algunas de las tasas más elevadas del mundo de niños con inseguridad alimentaria y malnutrición, se concentran ahora en zonas de conflicto.

Esto ha hecho saltar alarmas que no podemos ignorar: no acabaremos con el hambre y todas las formas de malnutrición para 2030 a menos que abordemos todos los factores que socavan la seguridad alimentaria y la nutrición. La hambruna golpeó diversas zonas de Sudán del Sur durante varios meses a principios de 2017, $\mathrm{y}$ existe un grave riesgo de que vuelva a producirse allí, así como de que aparezca en otros lugares afectados por conflictos, en 
especial el noreste de Nigeria, Somalia y Yemen.

El cambio climático, provocado por el aumento descontrolado de la emisión de gases de efecto invernadero, tiene consecuencias catastróficas para el planeta. El ciclo del agua se ha roto y el sistema de renovación no da abasto para renovar agua al ritmo que se consume, por lo que el control de los recursos hídricos se perfila igualmente como una de las fuentes principales de los futuros conflictos armados.

Estos procesos, y el mantenimiento de actividades de indudable riesgo ambiental, como la comercialización de nuevos productos químicos cuyos efectos a largo plazo no han sido investigados, la liberación de especies animales 0 vegetales genéticamente modificadas o la proliferación de la industria nuclear, afectan en mayor medida a los países empobrecidos.

Si partimos de que el $12 \%$ de la población mundial, que vive en Norteamérica y en Europa Occidental, realiza el $60 \%$ del consumo mundial, podemos entender la deuda ecológica como la deuda acumulada por los países industrializados hacia los países empobrecidos. Y, sin embargo, los países enriquecidos no hemos reflexionado $\mathrm{y}$ planteado seriamente los impactos medioambientales de la acción de expolio sistemático de recursos naturales; No han incorporado en su economía las externalidades que conlleva el actual modelo de desarrollo al no querer considerar los impactos medioambientales relacionados con la implantación de industrias en áreas no contaminadas como son los parques nacionales, las reservas naturales o los bosques amazónicos, por no citar lugares únicos con una biodiversidad extraordinaria.

La degradación ambiental contribuye además a ensanchar la brecha entre los diferentes grupos sociales al incidir directamente sobre las desigualdades, haciendo más evidentes los desequilibrios socio/económicos entre países o grupos sociales.

\section{PERO ¿CÓMO HEMOS LLEGADO HASTA AQUÍ?}

Para entender que un modelo de desarrollo, tan aparentemente poco equilibrado, se haya impuesto en todo el mundo como el más eficiente y racional, hay que remontarse a la etapa histórica en la que la ciencia se abre paso: La Modernidad.

En esta etapa, que culmina con la Ilustración a finales del S.XVIII, es cuando se desvincula el pensamiento del poder religioso y en el que el concepto de ciudadanía (exclusivamente masculina) se abre paso. Pero también es el Momento en el que despegó definitivamente el sistema capitalista, cuando se estableció la relación que debía darse entre los seres humanos y la naturaleza, y se creó un sistema tecno-científico que creció a unas velocidades incompatibles con los procesos que sostienen la vida.

La Modernidad se caracteriza por una estructura de pensamiento binario y jerarquizado, en el que la realidad se divide en una serie de dualismos, siendo uno de los más relevantes la oposición entre cultura y naturaleza. Así, el concepto del progreso humano se fue construyendo en oposición al estado de naturaleza, y en base al alejamiento de la misma.

El desarrollo tecnológico fue considerado como el motor del progreso al servicio de una idea simplificadora que asociaba consumo con bienestar $\mathrm{y}$, frente al saber tradicional, se impuso el conocimiento científico. Según este pensamiento, se consideró que todas las sociedades, de una forma lineal, evolucionaban de unos estadios de mayor "atraso" hacia nuevas etapas más racionales, de la caza y la 
recolección a la industrialización; y de la ausencia de propiedad privada a la economía de mercado.

La revolución científica condujo a conceptuar la naturaleza como una enorme máquina que podía ser diseccionada y estudiada por partes y a la que el ser humano, desde una posición externa a ella, podía dominar. Esta consideración de la naturaleza apuntaló una concepción antropocéntrica, según la cual los seres humanos se auto perciben como dueños de la naturaleza por "derecho divino".

Este antropocentrismo, que se materializó en la dominación del hombre sobre el resto del planeta, define todavía hoy, el comportamiento de la humanidad occidental, considerando a la Tierra como un enorme almacén al servicio de las personas.

Sin embargo, nuestra actual vulnerabilidad ante esta crisis ambiental no puede sino hacernos cuestionar esta supuesta independencia, para llevarnos a reconocer nuestra ecodependencia del mundo natural.

El último de los mitos, a mi entender, que arrastramos desde la Ilustración es el concepto de crecimiento, en el cuál el bienestar de las sociedades se encuentra ligado necesariamente al crecimiento económico. La economía de mercado tiene por objetivo la máxima utilidad de los factores de producción, obteniendo así beneficios que permiten acumular capital. Para maximizar el beneficio, los procesos que generan el mismo deben crecer también, a la vez que se minimizan los costes de producción. Así, en las últimas décadas, este proceso de crecimiento se ha multiplicado, y muchos países y empresas multinacionales han desarrollado formas de adquisición de recursos, materiales o humanos, cada vez más agresivas.

Ya hemos definido las consecuencias del conflicto entre un sistema económico, que necesita crecer, extraer materiales, fabricar cosas y generar residuos de forma constante y creciente para mantenerse, y un planeta con límites materiales y humanos. Por eso, podemos afirmar que el deterioro social y ambiental no es un daño colateral del modelo de desarrollo, sino que es una consecuencia ineludible de ese tipo de desarrollo.

$\mathrm{Si}$, como venimos diciendo, el sistema socioeconómico capitalista no funciona por haberse centrado en el mercado, el crecimiento y el beneficio individual, nuestra propuesta de transformación debe asentarse en el principio de "poner la vida en el centro", esto es, situar en el centro del modelo de desarrollo el sostenimiento de la vida y el bienestar colectivo.

Para poner remedio al pensamiento antropocéntrico y androcéntrico, necesitamos abrirnos a la diversidad y recoger, de la experiencia vivida por todos los seres humanos, aquellos conceptos que nos pueden ayudar a transformar la realidad para la sostenibilidad del planeta.

Mientras la ética de la justicia nos recuerda la obligación moral de no actuar injustamente con los otros, la empatía, habilidad necesaria para el siglo XXI, nos recuerda la obligación de no girar la cabeza ante las necesidades de los demás.

Por otro lado, frente al sentido materialista de la felicidad y bienestar construido por nuestras sociedades de consumo, resulta transformador enlazar con corrientes que defienden, no tanto un desarrollo alternativo, como una alternativa al desarrollo: "construir un nuevo paradigma que recupere el sentido de la vida desde la diversidad de opciones de pueblos y culturas". Esta visión de un desarrollo más sostenible y centrado en las personas, se aproxima al concepto de Desarrollo Humano definido por el programa de Naciones Unidas para el Desarrollo en 1990. Por otro lado, atajar el problema de sobrevelocidad que tenemos pasa 
por abandonar la obsesión intrínseca de este sistema por el crecimiento.

Significa que las sociedades tendremos que recortar de manera significativa nuestro consumo de recursos y producción de residuos hasta acoplarlos a la capacidad de producción y reciclaje de la naturaleza. El nuevo sistema debe celebrar la diversidad de todo lo vivo y superar visiones etnocéntricas que han conducido a la crisis ambiental y social. Existen vínculos que relacionan la diversidad genética, paisajística y cultural. La mayor densidad de bio-socio-diversidad se encuentra en la franja intertropical del planeta.

Es imprescindible ralentizar nuestra vida, nuestra forma de producir y consumir, de movernos. Hay que volver a acompasar nuestros ritmos con los del planeta. Una velocidad vital social alta implica una dificultad grande para ver por dónde y hacia dónde vamos, lo que restringe nuestra posibilidad de dirigir el camino adecuadamente. Desde las Universidades debemos, tenemos la responsabilidad social de reconducir nuestras acciones.

La búsqueda de ese nuevo modelo de desarrollo planetario requiere construir desde lo colectivo, se transpone en la vida social como una gestión democrática de las comunidades y sociedades, de manera que nos responsabilicemos de nuestros actos a través de la PARTICIPACIÓN SOCIAL. Y, cuando hablamos de democracia, nos referimos a una democracia participativa, en la que los valores básicos sean la cooperación, la horizontalidad, la equidad, la justicia, el biocentrismo (huyendo del antropocentrismo y el androcentrismo) y la libertad.

Un desarrollo que debe basarse en soluciones $y$ alternativas que busquen un equilibrio entre la igualdad social, un desarrollo económico equilibrado y un respeto a las limitaciones ambientales de los ecosistemas que componen la biosfera.

Nuestra supervivencia como especie depende de ello. La agenda 2030 y los 17 objetivos de desarrollo sostenible son la cuerda y el material que Naciones Unidas ha venido construyendo de forma participativa con todos los países y actores sociales para intentar superar tan altas cimas. Representan nuevas estrategias para hacer frente a viejos problemas, pero se ha aprendido de los errores cometidos en el pasado?

La aprobación en septiembre de 2015 de los nuevos Objetivos de Desarrollo Sostenible (ODS) y el Plan de Acción Global 2030 suponen un nuevo intento y cambio de referencia para mejorar la salud colectiva del Planeta. Se produce una evolución conceptual y de metodologías entre los ODM a los ODS en donde se integran objetivos sociales, ambientales y económicos dirigidos a todos los países, al Planeta en su globalidad.

Las universidades deberán actúan como modelos de referencia en nuestras sociedades, declaran y expanden su compromiso público por medio, principalmente, de una investigación responsable con los problemas ambientales y sociales de su entorno. Las instituciones de educación superior, empiezan a extender el valor y el impacto de sus enseñanzas e investigaciones a nivel local, actuando como catalizadores de cambio en sus comunidades más próximas. -La universidad como entidad docente e investigadora es un principal agente de cambio pues puede proporcionar respuestas a los problemas de la sociedad, experimentar científica y tecnológicamente las soluciones a dichos problemas y capacitar al capital humano que debe emprender el cambio (Alba, Benayas, 2006).

La educación para la sostenibilidad trata resolver la crisis civilizatoria en la que nos encontramos. 
La comunidad internacional está convencida de que se necesita desarrollar -a través de la educación- unos valores, comportamientos y modos de vida indispensables para un futuro sostenible y de que las universidades tienen un rol en este desafío. Así se han incorporado mandatos específicos para las universidades en las principales declaraciones de medio ambiente y sostenibilidad (Estocolmo 1972. Río 1992, Johannesburgo 2002), pero también se han elaborado y difundido diferentes declaraciones de sostenibilidad universitaria, a distintas escalas geográficas (Grindsted y Holm, 2012 y Lozano et al., 2013).

Aunque suene a evidencia, una universidad que promueva la sostenibilidad ha de ser sostenible y, para eso, han de ser transformadoras. La sostenibilidad ha de entrar a formar parte de los principios rectores de la actividad universitaria, ha de incorporarse al ADN de las universidades (Tilbury, 2010).

La responsabilidad social universitaria y la sostenibilidad ambiental deben entenderse como una reconceptualización del conjunto de la institución universitaria, a la luz de los valores, objetivos, formas de gestión e iniciativas que suponen un mayor compromiso con la sociedad y con la contribución a un modelo de desarrollo equilibrado y sostenible (Aznar, Ull y otros, 2014).

La nueva agenda 2030 y los nuevos ODS ofrecen una oportunidad única, pues integran en un mismo escenario de acción metas sociales, ambientales, económicas, de paz y alianzas. Los 17 objetivos consensuados a nivel mundial son un marco de trabajo que debería facilitar el camino y la búsqueda hacia un mundo más equitativo social y ambientalmente $\mathrm{y}$, consecuentemente, con un mayor nivel de bienestar humano. Se deberían incluir los contenidos y procedimientos (habilidades) para la sostenibilidad en todas las etapas educativas para romper con las inercias de los sucesivos currículos y materias. Sería conveniente seleccionar, y adaptar los ODS que se vayan a trabajar en cada curso como una manera de aumentar el interés del proceso de enseñanza-aprendizaje, favorecer el trabajo en equipo, y animar a la investigación educativa. Los ODS pueden servir como elemento transformador de planes de estudio y estándares de calidad nacionales y según señala Unesco: "Los planes de estudio tienen que garantizar que los futuros ciudadanosprofesionales aprendan no solo habilidades básicas, sino también habilidades transferibles, tales como el pensamiento crítico, la resolución de problemas, el activismo y la resolución de conflictos, para ayudarlos a convertirse en ciudadanos globales responsables".

Los estudiantes universitarios son los líderes del futuro, si perdemos la oportunidad de comprometerlos con los 17 retos de bienestar recogidos por la agenda 2030, estaremos perdiendo a toda una generación de referencia que será difícil de recuperar. La universidad debe hacer una apuesta clara por educar hacia opciones de futuro sostenible.

Las universidades como centros de investigación deberían primar e incentivar la realización de proyectos innovadores encaminados a alcanzar los ODS y una gestión más sostenible de los recursos. El futuro de la especie humana en el Planeta depende en gran medida de la capacidad que puedan tener las universidades y centros de investigación de generar opciones de consumo y forma de vida más sostenibles y viables. Nos encontramos en un momento crítico en el que se debe apostar por nuevos modelos de desarrollo y la universidad puede ser el laboratorio perfecto, no solo para idearlos y diseñarlos sino también para ponerlos en práctica y evaluar sus resultados. Las universidades deben ser los puntos neurálgicos de esa red de transformaciones que deben implantarse en toda la sociedad. 
La Responsabilidad Social Universitaria y la sostenibilidad deben entenderse como una RE CONCEPTUALIZACIÓN del conjunto de la Institución universitaria, a la luz de los valores, objetivos, formas de gestión en iniciativas que supongan un mayor compromiso con la sociedad $y$ con el futuro de las nuevas generaciones.

El concepto de la Responsabilidad Social y de la Sostenibilidad Universitaria se enmarca en un compromiso transversal, plural, progresivo y multidimensional, dentro del mundo universitario y de su proyección social.

La Responsabilidad Social y el Desarrollo Sostenible configuran una rol estratégico en la transferencia de conocimiento y de tecnología, combinando la innovación con la prosecución del bienestar, el dinamismo económico con la dimensión social.

La transferencia de conocimiento debe realizarse en un CONTEXTO SOCIALMENTE RESPONSABLE Y SOSTENIBLE.

El propósito de consolidar la Responsabilidad Social Universitaria y el Desarrollo Sostenible de las Universidades se fundamenta, además, en la concepción de estas instituciones como agente social, económico y cultural.

La Responsabilidad Social y la Sostenibilidad constituyen un eje central de la tercera Misión que la sociedad encomienda a la Universidad. El FOCO se centra en la contribución universitaria a un modelo de innovación y de desarrollo social, cultural, económico y ambiental socialmente responsable y sostenible.

ES UN COMPROMISO DE LAS INSTITUCIONES DE EDUCACIÓN SUPERIOR con las GENERACIONES ACTUALES Y VENIDERAS.

MUCHAS GRACIAS

\section{LITERATURA CITADA}

Alba, D., y Benayas, J., (2006) La universidad como referente social del cambio hacia un futuro sostenible. En Escolano, A., [Ed.] Educación superior y desarrollo sostenible. Discursos y prácticas. Biblioteca nueva. Madrid.

Aznar, P., Ull, M. A., Piñero, A., y MartínezAgut., M. P., (2014) La sostenibilidad en la formación universitaria: Desafios y oportunidades. Educación XXI, 17 (1), 133-158. https://doi.org/10.5944/educxx 1.17.1.10708

FAO, FIDA, OMS, PMA y UNICEF (2017). El estado de la seguridad alimentaria y la nutrición en el mundo 2017. Fomentando la resiliencia en aras de la paz y la seguridad alimentaria. Roma, FAO.

Fernández, R. (2011): La quiebra del capitalismo global 2000-2030, Libros en Acción, Baladre y Virus.

GREENPEACE, (2011). Tolerancia a herbicidas y cultivos transgénicos.

Grindsted, T. S. \& Holm, T., (2012) Thematic development of declarations on Sustainability in Higher Education. Environmental economics, Vol. 1. Issue, 1 Pp 32-39.https://doi.org/10.2139/ssrn.2 697500

GUNI Global University Network for Innovation, (2012) Higher Education in the World 4: Higher Education's Commitment to Sustainability from Understanding to Action. Pallgrave Macmillan.

Meadows, D. H.; Meadowns, D. L. Randers, J. y Beheren, W. W. (1972): Limits to growth, New York: Pontomac Associates. 
Sáenz, 2019

Orr, D., (1994) Earth in mind: On Education, Environment and the Human Prospect. Island. Press. Washington, D. C
Tilbury, D., (2010) Sustainability in the DNA of the university. Sustainable Mediterranean, 63-64. Pp. 9-13.

Copyright (c) 2019 Belén Sáenz-Rico de Santiago

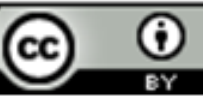

E ste tex to está protegido por una licencia CreativeCommons $\underline{4.0}$

Usted es libre para Compartir — copiar y redistribuir el $\mathrm{m}$ aterial en cualquier medio o formato- y Adaptar el documento —remezclar, transformar y crear a partir del material- para cualquier propósito, , incluso para fines com erciales, siempre que cumpla la condición de:

Atribución: Usted debe dar crédito a la obra original de manera adecuada, proporcionar un enlace a la licencia, e indicar si se han realizado cambios. Puede hacerlo en cualquier forma razonable, pero no de forma tal que sugiera que tiene el apoyo del licenciante o lo recibe por el uso que hace de la obra.

Resumendelicencia - Textocompletodelalicencia 